\title{
4)
}

\section{Securing accuracy and precision when pipetting hot and cold liquids with Microman ${ }^{\circledast}$}

\begin{abstract}
Biotechnology researchers and laboratory technicians cannot always prevent errors in their results owing to the temperature variation between samples and pipettes. Gilson, the pioneer in high-quality pipetting technology, has developed a positive displacement pipette, the Microman ${ }^{\oplus}$, to circumvent the issue. Here we present a comparison of Microman ${ }^{\circledR}$ performance with that of the Pipetman ${ }^{\circledR}$, the reliability standard from Gilson.
\end{abstract}

Observant scientists will have noticed that when they pipette cold samples, they invariably aspirate a larger-than-expected sample volume each time the tip is changed. Oddly, a smaller volume is delivered from the second aspiration onward if the tip is not changed. For example, pipetting hot samples, while they are cooling after their incubation at $37^{\circ} \mathrm{C}$, has the opposite effect.

Gilson conducted a study to determine the influence of sample temperature on the performance of Microman and Pipetman pipettes. Here we report the results and offer solutions to circumvent the issue. The work was performed in our ISO 17025-accredited laboratory.

\section{Testing protocol and observations}

We compared sample volumes in consecutive pipetting cycles of distilled water at $4{ }^{\circ} \mathrm{C}$ and $40{ }^{\circ} \mathrm{C}$ with volumes expected at $20^{\circ} \mathrm{C}$, the standard reference temperature for calibrating pipettes. We performed ten measurements each, without changing tips, of water at the two temperatures, using the Pipetman P100 with Gilson Diamond ${ }^{\circledR}$ tips and volume set at $100 \mu \mathrm{l}$ in a $20{ }^{\circ} \mathrm{C}$ regulated environment. When we aspirated a $4{ }^{\circ} \mathrm{C}$ sample for the first time, we observed that the dispensed volume was always greater than that obtained under standard conditions. Conversely, when we dispensed a $40{ }^{\circ} \mathrm{C}$ sample for the first time, the volume delivered was less than the reference. We also observed a sharp inversion of this phenomenon in both cases as of the second pipetting cycle (Fig. 1).

The first time water at $4{ }^{\circ} \mathrm{C}$ was aspirated, the temperature of the air cushion that is in contact with the water drops, causing the air

\section{Frédéric Millet \& Thierry Barthlen}

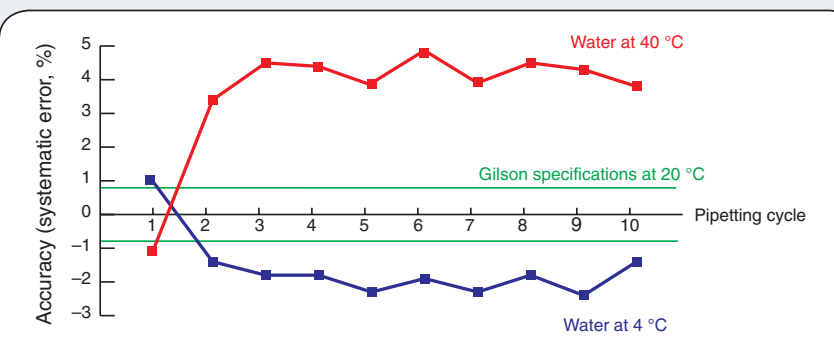

Figure 1 | An evaluation of the accuracy of a Pipetman P100 air displacement pipette as a function of sample temperature.

to shrink (Fig. 2). As the water and the air cushion share a fixed volume, the volume aspirated is higher than that under standard conditions $\left(\mathrm{V}_{\text {total }}=\mathrm{V}_{\text {air cushion }}+\right.$ sample $)$. During the second aspiration, the air cushion travels through a cold area, limited by the tip walls that were previously in contact with the cold sample, to a tepid region corresponding to the tip holder area that is never in contact with the liquid (Fig. 2). Consequently the air cushion temperature rises, causing the gas to expand. This is the opposite phenomenon of that observed during the first cycle.

The first time water at $40{ }^{\circ} \mathrm{C}$ is aspirated, the temperature of the air cushion that is in contact with the water rises causing the air to fill a greater volume. As a result, the sample volume dispensed is lower than what is expected under standard conditions. During the second aspiration, the air cushion travels from the warm area of the tip that was previously in contact with the water, to the tepid portion of the tip holder, which then causes a slight drop in the temperature and results in the air volume shrinking, producing a higher-thanexpected delivery volume.

From the third cycle onward, the delivered volume for cold samples stabilizes below the standard value (Fig. 2), and that for hot samples stabilizes above the standard value, but the volume 
APPLICATION NOTES

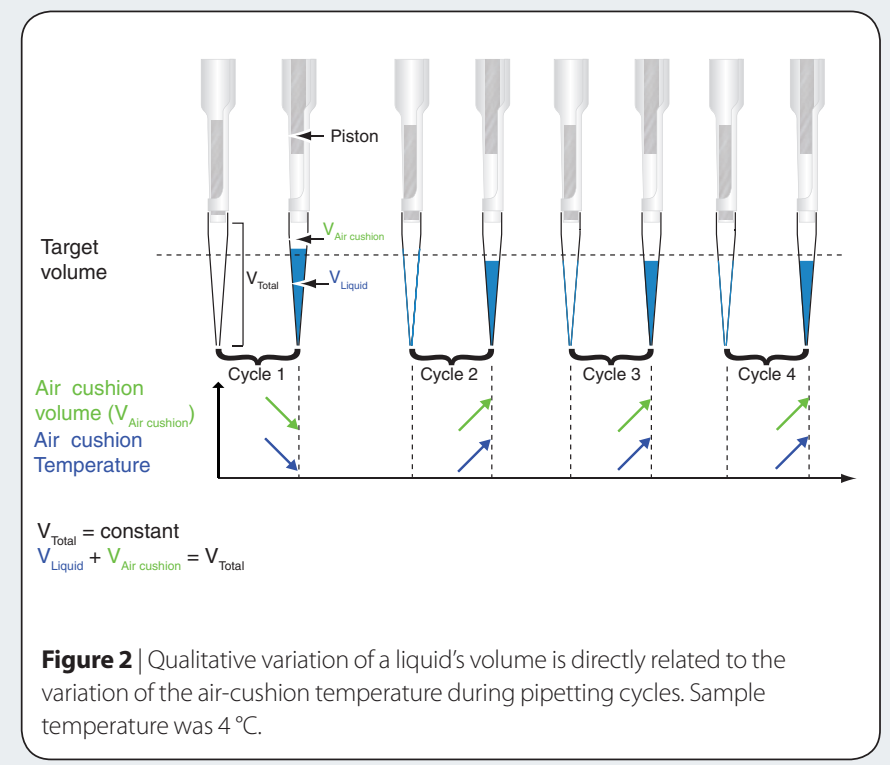

continues to fluctuate, albeit at a considerably lower rate. Reproducible results (limitation of random error) within the thresholds defined by the manufacturer's precision specifications cannot be obtained (Fig. 1). Our findings indicate that accuracy (systematic error) drifts by as much as $0.1-0.2 \%$ per degree of difference between the sample temperature and $20{ }^{\circ} \mathrm{C}$. The more the temperature gap widens, the greater the gravimetric errors become.

\section{Recommendations}

We recommend that liquids and pipettes be allowed to equilibrate to ambient temperature. If this is not possible, researchers can use certain techniques to offset the impact of temperature. One technique, prewetting, requires aspirating and dispensing the sample liquid three or four times before aspirating for sample delivery. The technique reduces the temperature difference between the sample and the air cushion, and it enhances precision without affecting accuracy. This produces results comparable to those of the second pipetting cycle.

Alternatively, we determined a theoretical correction factor that can be used to adjust the volume of Gilson Pipetman pipettes according to the sample temperature. The correction factor is $0.2 \%$

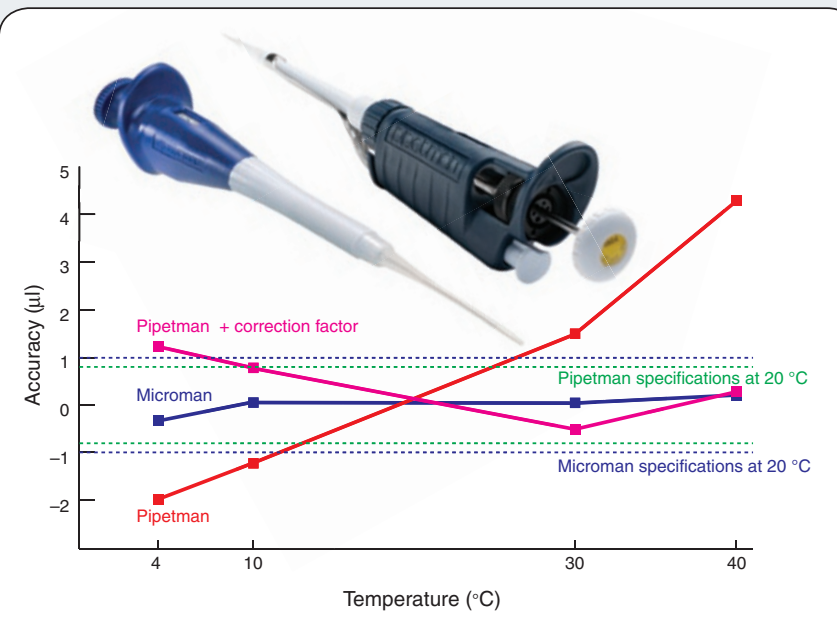

Figure 3 | Consistent accuracy and precision. Comparison of the accuracy (systematic error) of the Microman M100 positive-displacement pipette (left) and a Pipetman P100 air-displacement pipette (right) as a function of the sample temperature, with and without applying the correction factor.

per degree of difference between the sample temperature $\left(\mathrm{T}_{\text {sample }}\right)$ and $20{ }^{\circ} \mathrm{C}$. The volume setting can be calculated as follows:

$$
\begin{aligned}
& \text { Volume setting }=\text { target volume } \times\left(1-k \times\left(\mathrm{T}_{\text {sample }}-\mathrm{T}_{\text {room }}\right)\right), \\
& k=0.002{ }^{\circ} \mathrm{C}^{-1} .
\end{aligned}
$$

For example, to aspirate $100 \mu \mathrm{l}$ of a sample at $40{ }^{\circ} \mathrm{C}$ and room temperature at $20^{\circ} \mathrm{C}$, the volume setting should be $96 \mu \mathrm{l}$.

Researchers particularly concerned with data integrity can use positive displacement pipettes, which allow them to work accurately and precisely above or below the ambient temperature. The Gilson Microman ${ }^{\circledR}$ positive displacement pipette uses a disposable capillary and pistons, which isolate the aspirated sample from the inside parts of the pipette, eliminating the air space between the piston and sample. The benefit of using the Microman is that accuracy and precision remain consistent-whatever the sample temperature (Fig. 3)-eliminating the need for specific pipetting techniques.

This article was submitted to Nature Methods by a commercial organization and has not been peer reviewed. Nature Methods takes no responsibility for the accuracy or otherwise of the information provided. 